\title{
ON THE BEHAVIOUR OF HARMONIC FUNCTIONS AT THE BOUNDARY
}

\author{
BY \\ A. P. CALDERON( ${ }^{(1)}$
}

1. Let $F(x, y)$ be a function harmonic for $y>0$, and suppose that for every point $(x, 0)$ of a set $E$ of positive measure of the $x$-axis there exist a triangular region with vertex at that point, where the function is bounded; then it is well known that:

(A) Almost everywhere in $E, F(x, y)$ has a limit as $(x, y)$ tends nontangentially to $(x, 0) \in E$.

This result, first proved by Priwaloff $[1]\left({ }^{2}\right)$, when applied to analytic functions leads, as shown by Plessner [2], to the following stronger result:

(B) Let $F(z), z=x+i y$, be a function analytic for $y>0$. Then, except for $a$ set of points of measure zero, at every point $(x, 0)$ of the $x$-axis, either the function has a finite limit as $z$ tends nontangentially to $(x, 0)$, or the range of $F(z)$ in every triangular region with vertex at that point is dense in the whole complex plane.

Actually these results were proved not only for functions harmonic or analytic in a half-plane, but also in domains limited by rectifiable curves. However, even the special cases mentioned above were obtained by methods of conformal mapping [1], [2] which cannot be applied to harmonic or analytic functions of more variables.

A purpose of the present paper is to give a different proof of (A) which leads to its generalization to functions of any number of variables:

(a) Let $F(P), P=\left(x_{1}, x_{2}, \cdots, x_{n}\right)$, be a function harmonic for $x_{n}>0$ such that for every point $Q$ of a set $E$ of positive measure of the hyperplane $x_{n}=0$ there exists a region $\Gamma_{Q}$ limited by a cone with vertex at $Q$ and a hyperplane $x_{n}=$ const. where $F(P)$ is bounded. Then almost everywhere in $E$ the function has a limit as $P$ tends to $Q \in E$ nontangentially to $x_{n}=0$.

A further generalization of (A), which will enable us to extend (B) to functions of several complex variables, deals with functions which are harmonic in sets of variables, and may be stated as follows:

(b) Let $E=E_{1} \times E_{2} \times \cdots \times E_{m}$ be the Cartesian product of the spaces $E_{k}$ of points $P_{k}=\left(x_{1}^{(k)}, x_{2}^{(k)}, \cdots, x_{n}^{(k)}\right)$, and $F(P), P=\left(P_{1}, P_{2}, \cdots, P_{m}\right) \in E$, be defined and continuous in $x_{n}^{(k)}>0, k=1,2, \cdots, m$, and harmonic in $P_{k}$, that is, such that

Presented to the Society, September 2, 1949; received by the editors December 13, 1948, and, in revised form, June 20, 1949.

(1) Fellow of the Rockefeller Foundation.

(2) Numbers in brackets refer to the references cited at the end of the paper. 


$$
\sum_{i=1}^{n} \frac{\partial^{2} F}{\left(\partial x_{i}^{(k)}\right)^{2}}=0,
$$

$k=1,2, \cdots, m$.

Let $B_{k} \subset E_{k}$ be the space $x_{n}^{(k)}=0$, and $B=B_{1} \times B_{2} \times \cdots \times B_{m}$ the so-called distinguished boundary of $x_{n}^{(k)}>0, k=1,2, \cdots, m$, and suppose that for every point $Q=\left(Q_{1}, Q_{2}, \cdots, Q_{m}\right), Q_{i} \in B_{i}$, of a set $E$ of positive measure of $B$, there exist regions $\Gamma_{k Q}$, limited by cones with vertices at the points $Q_{k}$ and hyperplanes $x_{n}^{(k)}=$ const. such that the function $F(P)$ is bounded in $\Gamma_{Q}=\Gamma_{1 Q} \times \Gamma_{2 Q} \times \cdots \times \Gamma_{m Q}$. Then almost everywhere in $E, F(P)$ has a limit as $P=\left(P_{1}, P_{2}, \cdots, P_{m}\right)$ tends to $Q=\left(Q_{1}, Q_{2}, \cdots, Q_{m}\right) \in E$ in such a way that all $P_{k}$ tend to $Q_{k}$ simultaneously and nontangentially.

Once this is established it is not difficult to prove that:

(c) If $F\left(z_{1}, z_{2}, \cdots, z_{m}\right)$ is an analytic function of $z_{k}=x_{k}+i y_{k}$ regular for $y_{k}>0, k=1,2, \cdots, m$, then except for points belonging to a set of measure zero, at every point $Q=\left(x_{1}, x_{2}, \cdots, x_{m}\right)$ of the distinguished boundary $B, y_{1}=y_{2}$ $=\cdots=y_{m}=0$, either $F\left(z_{1}, z_{2}, \cdots, z_{m}\right)$ has a finite limit as all $z_{k}$ tend to $x_{k}$, simultaneously and nontangentially, or the range of the function in every region $\Gamma_{Q}=\Gamma_{1 Q} \times \Gamma_{2 Q} \times \cdots \times \Gamma_{m Q}$, product of triangular regions $\Gamma_{k Q}$ of the planes $z_{k}$ with vertices at $x_{k}$, is dense in the whole complex plane.

By means of inversions results similar to Theorem (a) can be obtained for functions harmonic inside or outside a sphere, or for functions like in (b) but defined in $E=E_{1} \times E_{2} \times \cdots \times E_{m}, E_{k}$ being either a half-space or the interior or exterior of a sphere. Also using the conformal mapping theorem of Riesz and Priwaloff [1], [3], (c) can be extended to functions $F\left(z_{1}, z_{2}, \cdots, z_{m}\right)$, analytic when $z_{k}$ belongs to a domain $D_{k}$ limited by a rectifiable curve.

Before passing to the proof of the results we wish to express our gratitude to Prof essor Antoni Zygmund, who proposed the present topic and helped us with valuable suggestions.

2. In this section we shall prove (b). The proposition (a) is contained in (b) as a special case and therefore we shall omit its proof.

However, if one specializes suitably the following argument, (a) can be established directly.

For the sake of simplicity we shall confine ourselves to the special case in which $E=E_{1} \times E_{2}, E_{1}$ and $E_{2}$ being the three-dimensional spaces of points $P_{1}=\left(x_{1}, y_{1}, z_{1}\right)$ and $P_{2}=\left(x_{2}, y_{2}, z_{2}\right)$ respectively, the same argument applying mutatis mutandis to the general case. We have then a function $F(P)$, $P=\left(P_{1}, P_{2}\right)$, harmonic in $P_{1}$ and $P_{2}$ for $z_{1}>0, z_{2}>0$, such that at every point $Q=\left(Q_{1}, Q_{2}\right)$ of a set $E$ of positive measure of $z_{1}=z_{2}=0$ there exist two regions, $\Gamma_{1 Q}$ and $\Gamma_{2 Q}$ limited by cones with vertices at $Q_{1}$ and $Q_{2}$ and planes $z_{1}=$ const. and $z_{2}=$ const., such that $F(P)$ is bounded in $\Gamma_{Q}=\Gamma_{1 Q} \times \Gamma_{2 Q}$. First we shall show that from the beginning we may make a stronger assumption about the regions $\Gamma_{Q}$. We may assume in fact that for $0<z_{1}<h$ and $0<z_{2}<h, F(P)$ is uniformly bounded in all regions $\gamma_{Q}=\gamma_{Q_{1}} \times \gamma_{Q_{2}}, Q=\left(Q_{1}, Q_{2}\right) \in E, \gamma_{Q_{1}}$ and 
$\gamma_{Q_{2}}$ being the cones with vertices at $Q_{1}$ and $Q_{2}$ respectively, and parallel to $\alpha^{2} z^{2}>x^{2}+y^{2}, z>0, \alpha$ being any fixed positive number. This will be a consequence of the following lemma.

LEMma. If $F(P)$ is a function continuous for $z_{1}>0, z_{2}>0$, and for every point $Q$ belonging to a set $E$ of positive measure of $z_{1}=z_{2}=0$ the function is bounded in a region $\Gamma_{Q}$, then, given any $\alpha$ and $\epsilon>0$, there exist a constant $h>0$ and $a$ set $E^{\prime}, E^{\prime} \subset E,\left|E^{\prime}\right|>|E|-\epsilon$, such that for $0<z_{1}<h, 0<z_{2}<h$, the function is uniformly bounded in all $\gamma_{Q}, Q \in E^{\prime}$.

It is clear that there exists a sequence of regions $\Gamma^{(n)}$, limited by cones and planes $z=$ const., such that every region $\Gamma_{1 Q}$ or $\Gamma_{2 Q}$ contains a conical region with the same vertex and congruent to some $\Gamma^{(n)}$. Let $\Gamma_{1 Q}^{(n)}$ be the region congruent to $\Gamma^{(n)}$ with vertex at $Q_{1}$, and $\Gamma_{2 Q}^{(n)}$ the region congruent to $\Gamma^{(n)}$ with vertex at $Q_{2}$. Let $E_{n m r}$ denote the set of points $Q$ of $z_{1}=z_{2}=0$ such that $|F(P)|<r$ in $\Gamma_{1 Q}^{(n)} \times \Gamma_{2 Q}^{(m)}$; since $F(P)$ is continuous, $E_{n m r}$ is measurable and since every point of $E$ belongs to some $E_{n m r}$, we have $E \subset \cup E_{n m r}$. Let now $\bar{Q}$ be a point of strong density $\left(^{3}\right)$ of $E_{n m r}$; then every point $P=\left(P_{1}, P_{2}\right)$ of $\gamma_{\bar{Q}}$ belongs to some $\Gamma_{1 Q}^{(n)} \times \Gamma_{1 Q}^{(m)}, Q \in E_{n m r}$, for $z_{1}$ and $z_{2}$ small enough. To show it, draw with vertices at $P_{1}$ and $P_{2}$ two cones parallel to those of $\Gamma^{(n)}$ and $\Gamma^{(m)}$ respectively and directed towards the planes $z_{1}=0$ and $z_{2}=0$, which they intersect in two ellipses $\Sigma_{1}$ and $\Sigma_{2}$; for $P_{1}$ and $P_{2}$ close enough to $z_{1}=0$ and $z_{2}=0$, $P=\left(P_{1}, P_{2}\right)$ is contained in $\Gamma_{1 Q}^{(n)} \times \Gamma_{2 Q}^{(m)}, Q=\left(Q_{1}, Q_{2}\right) \in E_{n m r}$, if $Q_{1} \in \Sigma_{1}$ and $Q_{2} \in \Sigma_{2}$, that is, if $\Sigma_{1} \times \Sigma_{2}$ contains the point $Q$ of $E_{n m r}$; but since $\bar{Q}$ is a point of strong density of $E_{n m r}$, the average density of this set in $\Sigma_{1} \times \Sigma_{2}$ tends to one as $P$ tends to $\bar{Q}$ remaining inside $\gamma_{\bar{Q}}$ and $\Sigma_{1} \times \Sigma_{2}$ certainly contains a point of $E_{n m r}$ for $z_{1}$ and $z_{2}$ small enough. Hence if we denote by $E_{n}$ the set of points $Q$ such that $|F(P)|<n$ in $\gamma_{Q}, Q \in E_{n}$, for $0<z_{1}<1 / n$ and $0<z_{2}<1 / n$, then except for a set of measure zero, every point of $E_{n m r}$ belongs to some $E_{n}$, and since $E \subset \cup E_{n m r}$, the same thing holds for $E$; that is, except for a set of measure zero, every point of $E$ is contained in $\cup_{1}^{\infty} E_{n}$, and so $\left|\cup_{1}^{\infty} E_{n}\right| \geqq|E|$. But the sets $E_{n}$ are increasing, that is, $E_{1} \subseteq E_{2}$ $\subseteq \cdots$; hence $\lim _{n \rightarrow \infty}\left|E_{n}\right|=\left|\cup E_{n}\right| \geqq|E|$, and $n \geqq n_{0}$, we have $\left|E_{n}\right|$ $\geqq|E|-\epsilon$, which proves the lemma. This shows that if we prove the theorem under the stronger assumption that the set $E$ is closed and the function is uniformly bounded in all regions $\gamma_{Q}, Q \in E$, for $0<z_{1}<h$ and $0<z_{2}<h$, the existence of the limit will be established for subsets of measures arbitrarily close to that of the given set, that is, for the given set itself. Hence, returning

(3) We use here the strong density theorem in a form slightly more general than the usual one. Let $Q$ be a point of a set $E$ and $C$ any domain such that the quotient of its measure by the measure of the smallest interval with edges parallel to the axis and containing both $Q$ and $C$ exceeds a fixed positive number. Then, as remarked to us by Professor Zygmund, the theorem still holds, that is, for almost all $Q$ of $E$, the average density of $E$ in $C$ tends to one as the largest edge of the interval tends to zero, and the result is a simple consequence of the strong density theorem in its usual form. 
to our function $F(P)$, we shall assume that, for $0<z_{1}<2$ and $0<z_{2}<2$, $|F(P)|<1$ in all $\gamma_{Q}, Q$ belonging to a closed set $E$ of positive measure. We shall also assume that in the cone $\alpha^{2} z^{2}>x^{2}+y^{2}$ we have $\alpha=1$.

Let $\bar{D}$ be the region of all points of $\bigcup_{Q \in E} \gamma_{Q}$ contained in $0<z_{1}<2$, $0<z_{2}<2$, and $D$ that of all points contained in $0<z_{1}<1,0<z_{2}<1$. Let $F_{n}(P)$ $=F\left(x_{1}, y_{1}, z_{1}+1 / n, x_{2}, y_{2}, z_{2}+1 / n\right)$ and $D_{n}$ be the domains obtained by shifting $\bar{D}$ by $-1 / n$ in the directions of the $z_{1}$ and $z_{2}$ axes. We have $D \subset D_{n} ;\left|F_{n}(P)\right|<1$ in $D_{n}$ and continuous in $z_{1} \geqq 0, z_{2} \geqq 0$. If $B$ denotes as before the distinguished boundary $z_{1}=z_{2}=0$ and because $D_{n}$ is an open set, we have $D_{n} \cap B$ is open in $B$ and $E \subset D_{n} \cap B$. Let now $F_{n}^{*}$ be a function continuous and less than 1 in absolute value, equal to $F_{n}$ in $E$ and vanishing outside $D_{n} \cap B$, and let

$$
\phi_{n}\left(P_{1}, P_{2}\right)=\frac{1}{4 \pi^{2}} \int_{B} \frac{z_{1}}{\left|P_{1}-Q_{1}\right|^{3}} \frac{z_{2}}{\left|P_{2}-Q_{2}\right|^{3}} F_{n}^{*}\left(Q_{1}, Q_{2}\right) d \sigma_{1} d \sigma_{2}
$$

where $\left|P_{1}-Q_{1}\right|$ and $\left|P_{2}-Q_{2}\right|$ are the distances between $P_{1}$ and $Q_{1}$ and $P_{2}$ and $Q_{2}$ respectively; $z_{1}$ and $z_{2}$ are the coordinates of $P_{1}$ and $P_{2}$; and $d \sigma_{1}$ and $d \sigma_{2}$ are the elements of area of $z_{1}=0$ and $z_{2}=0$ respectively. The function $\phi_{n}\left(P_{1}, P_{2}\right)$ is the double Poisson integral of the function $F_{n}^{*}\left(Q_{1}, Q_{2}\right)$, and is therefore a function harmonic in $\left(x_{1}, y_{1}, z_{1}\right)$ and $\left(x_{2}, y_{2}, z_{2}\right)$ separately. Since $F_{n}{ }^{*}\left(Q_{1}, Q_{2}\right)$ is continuous in $B$ and its absolute value is less than 1 there, $\phi_{n}\left(P_{1}, P_{2}\right)$ is continuous in $D_{n}$ for $z_{1} \geqq 0$ and $z_{2} \geqq 0, \phi_{n}=F_{n}^{*}$ in $B$ and $\left|\phi_{n}\left(P_{1}, P_{2}\right)\right|<1$. Hence the functions $\psi_{n}=F_{n}-\phi_{n}$ are continuous in $D_{n}$ for $z_{1} \geqq 0$ and $z_{2} \geqq 0$, vanish in $E$, because $\phi_{n}=F_{n}^{*}=F_{n}$ there, and $\left|\psi_{n}\right|<2$ in $D_{n}$. Because $\left|\phi_{n}\right|<1$ we may select a subsequence $\phi_{n_{i}}$ converging to a function $\phi\left(P_{1}, P_{2}\right)$ harmonic in $P_{1}$ and $P_{2}$ separately, and since $F_{n}$ converges to $F, \psi_{n_{i}}$ will converge to a function $\psi$ and we have finally $F=\phi+\psi$. Now $\phi$ is in absolute value less than 1 in $z_{1}>0, z_{2}>0$ and may therefore be represented by a double Poisson integral

$$
\phi\left(P_{1}, P_{2}\right)=\frac{1}{4 \pi^{2}} \int_{B} \frac{z_{1}}{\left|P_{1}-Q_{1}\right|^{3}} \frac{z_{2}}{\left|P_{2}-Q_{2}\right|^{3}} \phi\left(Q_{1}, Q_{2}\right) d \sigma_{1} d \sigma_{2},
$$

$\phi\left(Q_{1}, Q_{2}\right)$ being a bounded measurable function, and therefore $\phi\left({ }^{4}\right)$ has a limit as $P=\left(P_{1}, P_{2}\right)$ tends to $Q=\left(Q_{1}, Q_{2}\right)$ in such a way that $P_{1}$ tends to $Q_{1}$ and $P_{2}$ to $Q_{2}$ simultaneously and nontangentially to $z_{1}=0$ and $z_{2}=0$, almost everywhere in $B$. It remains then to show that also $\psi$ has a limit almost everywhere in $E$. Now $\psi$ is the limit of functions $\psi_{n_{i}}$ which are all continuous and vanishing in $E$. Therefore it is natural to expect that $\psi$ will tend to zero almost everywhere in $E$. To prove this we shall construct a function $\Omega\left(P_{1}, P_{2}\right)$ positive and harmonic in $D$, tending to zero almost everywhere in $E$ and such that at every boundary point of $D$ the inequalities

(4) See [4]. The result is stated there for the case of a bicylinder and of radial approach only, but the proof (a simple consequence of strong differentiability of the integral of a bounded function) is perfectly general. 


$$
-2 \Omega\left(P_{1}, P_{2}\right) \leqq \psi_{n}\left(P_{1}, P_{2}\right) \leqq 2 \Omega\left(P_{1}, P_{2}\right)
$$

will hold. Now applying the principle of maximum the inequalities extend to the whole domain $D$, and passing then to the limit we shall obtain

$$
-2 \Omega\left(P_{1}, P_{2}\right) \leqq \psi\left(P_{1}, P_{2}\right) \leqq 2 \Omega\left(P_{1}, P_{2}\right) \text {. }
$$

This will prove that $\psi$ tends to zero as $\left(P_{1}, P_{2}\right)$ tends to $\left(Q_{1}, Q_{2}\right) \in E$, along any path contained in $D$, almost everywhere in $E$; now the restriction on the path is only apparent, because in virtue of the lemma, for all $Q \in E$ not belonging to a set of measure zero, any path ending at $Q$ and such that its projections on $E_{1}$ and $E_{2}$ are nontangential to $z_{1}=0$ and $z_{2}=0$ is contained in $D$ for $z$ and $z_{2}$ small enough.

Let us now pass to the construction of the function $\Omega\left(P_{1}, P_{2}\right)$. Let $C E$ be the set complementary to $E$ in $B$ and $c\left(Q_{1}, Q_{2}\right)$ its characteristic function. We define

$$
\Omega\left(P_{1}, P_{2}\right)=z_{1}+z_{2}+\int_{B} \frac{z_{1}}{\left|P_{1}-Q_{1}\right|^{3}} \frac{z_{2}}{\left|P_{2}-Q_{2}\right|^{3}} c\left(Q_{1}, Q_{2}\right) d \sigma_{1} d \sigma_{2} .
$$

This function is positive, harmonic in $P_{1}$ and $P_{2}$ and tends to zero almost everywhere in $E$. We shall investigate the behaviour of $\Omega$ at the boundary of $D$.

Let first $\bar{P}=\left(\bar{P}_{1}, \bar{P}_{2}\right)$ be a point of the boundary of $D$ for which $z_{1}$ or $z_{2}$ is equal to 1 , then $\Omega\left(\bar{P}_{1}, \bar{P}_{2}\right) \geqq 1$. Let now $\bar{P}=\left(\bar{P}_{1}, \bar{P}_{2}\right)$ be a boundary point of $D$ such that $0<z_{1}<1$ and $0<z_{2}<1$, then $\Omega\left(\bar{P}_{1}, \bar{P}_{2}\right)$ exceeds 1 . In fact, let us draw with vertices at $P_{1}$ and $P_{2}$ two cones parallel to $z^{2}=x^{2}+y^{2}$ and directed downwards; they meet $z_{1}=0$ and $z_{2}=0$ in two circles $\Sigma_{1}$ and $\Sigma_{2} ; \Sigma_{1} \times \Sigma_{2}$ contains no point of $E$ in its interior, for otherwise $\left(\bar{P}_{1}, \bar{P}_{2}\right)$ would be contained in $D$. Hence $c\left(Q_{1}, Q_{2}\right)$ is equal to one in $\Sigma_{1} \times \Sigma_{2}$ and we have

$$
\Omega\left(\bar{P}_{1}, \bar{P}_{2}\right) \geqq \int_{\Sigma_{1} \times \Sigma_{2}} \frac{z_{1}}{\left|\bar{P}_{1}-Q_{1}\right|^{3}} \frac{z_{2}}{\left|\bar{P}_{2}-Q_{2}\right|^{3}} d \sigma_{1} d \sigma_{2}
$$

Computing this integral in polar coordinates we obtain

$$
\Omega\left(\bar{P}_{1}, \bar{P}_{2}\right) \geqq\left[\int_{0}^{z_{1}} \frac{z_{1}}{\left(\rho^{2}+z_{1}^{2}\right)^{3 / 2}} 2 \pi \rho d \rho\right]\left[\int_{0}^{z_{2}} \frac{z_{2}}{\left(\rho^{2}+z_{2}^{2}\right)^{3 / 2}} 2 \pi \rho d \rho\right]
$$

and finally, introducing the variables $\rho=z_{1} s$ and $\rho=z_{2} s$,

$$
\Omega\left(\bar{P}_{1}, \bar{P}_{2}\right) \geqq\left[4 \pi^{2} \int_{0}^{1} \frac{s d s}{\left(1+s^{2}\right)^{3 / 2}}\right]>1 \text {. }
$$

Finally let $\bar{P}=\left(\bar{P}_{1}, \bar{P}_{2}\right)$ be a point such that $z_{1}>0$ and $z_{2}=0$; that is, $\bar{P}_{2}$ coincides with a point $\bar{Q}_{2}$ of $z_{2}=0$. The function $\Omega$ is not defined there, but we may estimate the lower limit of $\Omega\left(P_{1}, P_{2}\right)$ as $\left(P_{1}, P_{2}\right)$ tends to $\left(\bar{P}_{1}, \bar{P}_{2}\right)$. By (1) we 
have

$$
\Omega\left(P_{1}, P_{2}\right) \geqq z_{1}+2 \pi \int_{B_{1}} \frac{z_{1}}{\left|P_{1}-Q_{1}\right|^{3}}\left[\frac{1}{2 \pi} \int_{B_{2}} \frac{z_{2}}{\left|P_{2}-Q_{2}\right|^{3}} c\left(Q_{1}, Q_{2}\right) d \sigma_{2}\right] d \sigma_{1},
$$

but $c\left(Q_{1}, Q_{2}\right)$ is the characteristic function of an open set, and so is continuous at every point where it is equal to one; hence the inner integral above tends to $c\left(Q_{1}, \bar{Q}_{2}\right)$ whenever $c\left(Q_{1}, \bar{Q}_{2}\right)=1$; at the other points $c\left(Q_{1}, \bar{Q}_{2}\right)$ is zero, and the integral being non-negative its lower limit is also non-negative; that is, $c\left(Q_{1} \bar{Q}_{2}\right)$ never exceeds the lower limit of the integral and hence

$$
\lim \inf \Omega\left(P_{1}, P_{2}\right) \geqq z_{1}+2 \pi \int_{B_{1}} \frac{z_{1}}{\left|P_{1}-Q_{1}\right|^{3}} c\left(Q_{1}, \bar{Q}_{2}\right) d \sigma,
$$

as $\left(P_{1}, P_{2}\right)$ tends to $\left(\bar{P}_{1}, \bar{Q}_{2}\right)$. Let us denote the right-hand side of the last inequality by $\omega\left(\bar{P}_{1}, \bar{Q}_{2}\right)$, and by $D_{\bar{Q}_{2}}$ the set of all points $\left(P_{1}, \bar{Q}_{2}\right), 0<z_{1}<1$, such that $P_{1}$ is contained in some cone parallel to $z^{2}>x^{2}+y^{2}$ with vertex at $Q_{1}$, $\left(Q_{1}, \bar{Q}_{2}\right) \in E$. The function $\omega\left(\bar{P}_{1}, \bar{Q}_{2}\right)$ of $\bar{P}_{1}$ is quite similar to $\Omega\left(P_{1}, P_{2}\right)$; it is harmonic in $\bar{P}_{1}$ non-negative, and exceeds 1 at every point not contained in $D_{\bar{Q} \mathbf{2}}$ for which $z_{1}>0$. The proof of this property is the same as for $\Omega$ and therefore we omit it.

We are now in a position to show that we can majorize the functions $\psi_{n}$ by $\Omega$ in $D$. More precisely we shall prove that at every boundary point of $D$ either

$$
-2 \Omega \leqq \psi_{n} \leqq 2 \Omega
$$

or

$$
-(\lim \inf 2 \Omega) \leqq \psi_{n} \leqq \lim \inf 2 \Omega
$$

holds, and so, in virtue of the principle of maximum, the validity of the first inequalities throughout $D$ will follow and the proof of the theorem will be complete.

First let $\left(\bar{P}_{1}, \bar{P}_{2}\right)$ be a boundary point of $D$ for which $z_{1}>0$ and $z_{2}>0$ since $\left|\psi_{n}\right|<2$ in $D_{n}$ and $\Omega \geqq 1$ at this point, the inequality (2) follows in this case.

Let now $\left(\bar{P}_{1}, \bar{Q}_{2}\right)$ be a boundary point such that $z_{1}>0, z_{2}=0$. Consider the function $2 \Omega-\psi_{n}$; we have to prove that

$$
\lim \inf \left(2 \Omega-\psi_{n}\right) \geqq 0 .
$$

If $\left(\bar{P}_{1}, \bar{Q}_{2}\right)$ is not contained in $D_{\bar{Q}_{2}}$, we know that lim inf $2 \Omega \geqq 2 \omega\left(\bar{P}_{1}, \bar{Q}_{2}\right) \geqq 2$ and since $\left|\psi_{n}\right|<2$ in $D$, we have the inequality above. If $\left(\bar{P}_{1}, \bar{Q}_{2}\right)$ is contained in $D_{\bar{Q}_{2}}$, it is also contained in $D_{n}$, as is easily seen by the definitions of $D_{\bar{Q}_{2}}$ and $D_{n}$. Now $\psi_{n}$ is a function continuous in $D_{n}$ for $z_{1} \geqq 0$ and $z_{2} \geqq 0$, and so $\psi_{n}\left(P_{1}, P_{2}\right)$ tends to $\psi_{n}\left(\bar{P}_{1}, \bar{Q}_{2}\right)$ as $\left(P_{1}, P_{2}\right)$ tends to $\left(\bar{P}_{1}, \bar{Q}_{2}\right)$. Hence

$$
\lim \inf \left(2 \Omega-\psi_{n}\right) \geqq 2 \omega\left(\bar{P}_{1}, \bar{Q}_{2}\right)-\psi_{n}\left(\bar{P}_{1}, \bar{Q}_{2}\right) \text {. }
$$


Now $\psi_{n}\left(\bar{P}_{1}, \bar{Q}_{2}\right)$ is a continuous function of $\bar{P}_{1}$ in $D_{\bar{Q}_{2}}$, and being the limit of functions $\psi_{n}\left(P_{1}, P_{2}\right)$ harmonic in $P_{1}$ and uniformly bounded, it is also harmonic in $\bar{P}_{1}$. Moreover, we have $\left|\psi_{n}\right|<2$ in $D_{n}$ and hence $\left|\psi_{n}\right|<2$ in $D_{\bar{Q}_{2}}$; and finally, as $\bar{P}_{1}$ tends to a point $Q_{1}\left(Q_{1}, \bar{Q}_{2}\right) \in E, \psi_{n}\left(\bar{P}_{1}, \bar{Q}_{2}\right)$ tends to zero because $\psi_{n}$ is continuous and vanishes in $E$. From this and from the properties of $\omega\left(\bar{P}_{1}, \bar{Q}_{2}\right)$ we stated above, it follows that the function of $\bar{P}$

$$
2 \omega\left(\bar{P}_{1}, \bar{Q}_{2}\right)-\psi_{n}\left(\bar{P}_{1}, \bar{Q}_{2}\right)
$$

is non-negative, or has a non-negative lower limit at every boundary point of $D \bar{Q}_{2}$, and so, by the harmonicity of the function in $\bar{P}_{1}$, and in virtue of the principle of maximum, we have

$$
2 \omega\left(\bar{P}_{1}, \bar{Q}_{1}\right)-\psi_{n}\left(\bar{P}_{1}, \bar{Q}_{2}\right) \geqq 0
$$

in $D_{\bar{Q}_{2}} ;$ and in virtue of (4)

$$
\lim \inf \left(2 \Omega-\psi_{n}\right) \geqq 0
$$

when $\left(P_{1}, P_{2}\right)$ tends to any boundary point $\left(\bar{P}_{1}, \bar{Q}_{2}\right)$ of $D$.

The case in which for the boundary point we have $z_{1}=0, z_{2}>0$, that is, $\bar{P}_{1}$ coincides with a point $\bar{Q}_{1}$ of $z_{1}=0$, may be treated in exactly the same way as the preceding.

Finally suppose that $\left(\bar{P}_{1}, \bar{P}_{2}\right)$ is a boundary point of $D$ such that $z_{1}=z_{2}=0$. Then $\left(\bar{P}_{1}, \bar{P}_{2}\right) \in E$ and since $\psi_{n}$ is continuous and vanishes in $E$ and $\Omega$ is non-negative we have again $\lim \inf \left(2 \Omega-\psi_{n}\right) \geqq 0$. Hence the proof of the righthand side of ( 3 ) is complete.

Arguing in the same way as above on $-\psi_{n}$, the left-hand side of (3) follows, and the theorem is established.

Finally we need remark that the foregoing argument still holds if we assume that $F(P)$ is defined only in the domain $\bar{D}$, or in the regions $\Gamma_{Q}$. This fact will be needed in the proof of (c).

3. To establish Theorem (c) we shall use the same technique used by Plessner in the proof of his result.

Let $F\left(z_{1}, z_{2}, \cdots, z_{m}\right), z_{k}=x_{k}+i y_{k}$, be an analytic function regular in $y_{k}>0, k=1,2, \cdots, m$. Consider the sequence $\left\{C_{n}\right\}$ of all circles of the complex plane with rational radii and centers $w_{n}$ with rational coordinates, and let $E_{n}$ be the set of points $Q$ of the distinguished boundary $B, y_{1}=y_{2}=\cdots=y_{m}=0$, for which there exists a region $\Gamma_{Q}=\Gamma_{1 Q} \times \Gamma_{2 Q} \times \cdots \times \Gamma_{m Q}$ where the function does not take values of $C_{n}$. The continuity of $F\left(z_{1}, z_{2}, \cdots, z_{m}\right)$ in $y_{k}>0$ implies that the sets are $E_{n}$ measurable. Let now $E$ be the union of all sets $E_{n}$, and $C E$ the set complementary to $E$ in $B$; then in every region $\Gamma_{Q}=\Gamma_{1 Q} \times \cdots$ $\times \Gamma_{m Q}, Q \in C E$, the range of the function is dense in the whole complex plane, for otherwise $F\left(z_{1}, z_{2}, \cdots, z_{m}\right)$ would not take values of some $C_{n}$ in $\Gamma_{Q}$, and hence $Q$ would belong to some $E_{n}$. Now, at every point $Q$ of $E_{n}$ the function does not take values of $C_{n}$ in $\Gamma_{Q}$, and so $\phi=\left(F-w_{n}\right)^{-1}$ is a function bounded 
in all $\Gamma_{Q}, Q \in E_{n}$. But this function is analytic in $z_{k}, k=1,2, \cdots, m$, that is, harmonic in the pairs of variables $\left(x_{k}, y_{k}\right)$, and so, by Theorem (b), has a limit as $\left(z_{1}, z_{2}, \cdots, z_{m}\right)$ tends to $Q$ nontangentially to $y_{k}=0$ almost everywhere in $E_{n}$. Hence $F=w_{n}+\phi^{-1}$ has also a finite or infinite limit almost everywhere in $E_{n}$; and since the same is true for all $E_{n}, F$ has a finite or infinite limit almost everywhere in $E$. It remains only to show that this limit must be finite almost everywhere.

In order to prove this let us consider the function

$$
\phi(z)=F\left(\alpha_{1}+z, \alpha_{2}+z, \cdots, \alpha_{m}+z\right),
$$

$z=x+i y$, the $\alpha_{k}$ being real parameters. Let $E^{*}$ be the subset of $E$ where the limit of $F\left(z_{1}, z_{2}, \cdots, z_{m}\right)$ is infinite, and $x_{k}=\alpha_{k}+t, k=1,2, \cdots, m$, a straight line of the space $B$ of variables $x_{k}$. If $\bar{x}_{k}=\alpha_{k}+\bar{t}$ is a point of intersection of the line with $E^{*}$ we have evidently $\lim \phi(z)=\infty$ as $z \rightarrow \bar{t}$ nontangentially to $y=0$. But according to (B) this can happen only for a set of measure zero of $y=0$, that is, for a set of measure zero of values of $t$. Hence the line intersects $E^{*}$ in a set of measure zero, and, since the same holds for any values of the parameters $\alpha_{k}$, every straight line meets $E^{*}$ in a set of measure zero. But, $E^{*}$ being measurable, this is possible only if its measure is zero.

This completes the proof of (c).

\section{REFERENCES}

1. I. Priwaloff, Sur une généralisation du théorème de Fatou, Rec. Math. (Mat. Sbornik) vol. 31 (1923) pp. 232-235.

2. A. Plessner, Ueber das Verhalten analytischer Funktionen auf dem Rande des Definitionsbereiches, J. Reine Angew. Math. vol. 158 (1928) pp. 219-227.

3. F. Riesz and M. Riesz, Ueber die Randwerte einer analytischer Funktion, Math. Zeit. vol. 18 (1922) pp. 87-95.

4. A. Zygmund, On the differentiability of multiple integrals, Fund. Math. vol. 23 (1934) pp. 143-149 (Corrigenda, ibid. vol. 25 (1935) p. 234).

University of Chicago,

Chicago, Ill. 